\title{
VENTAJAS ORGANIZACIONALES, ECONÓMICAS Y SOCIALES, DERIVADAS DE LA APLICACIÓN DE LA SUBROGACIÓN DE PERSONAL EN EL SECTOR DE LA SEGURIDAD PRIVADA EN ESPAÑA
}

\section{ORGANIZATIONAL, ECONOMIC AND SOCIAL ADVANTAGES, DERIVED FROM THE APPLICATION OF SUBROGATION OF PERSONNEL IN THE PRIVATE SECURITY SECTOR IN SPAIN}

Santiago Ávila Vila (EAE Business School) ${ }^{1}$

Ana Belén Campuzano Laguillo (Universidad CEU San Pablo) ${ }^{2}$

Marcelo Pascual Faura (Universidad CEU San Pablo) ${ }^{3}$

\section{Resumen:}

Desde finales del siglo pasado, el sector de la seguridad privada en España se ha concretado como un agente económico y social de relevancia, lo que se deriva del impacto que tiene su volumen de negocio en la economía del país y de la gran cantidad de empleo estable que propicia la figura jurídica de la subrogación de personal. El objetivo de este trabajo se centra en dar respuesta a dos cuestiones básicas sobre la aplicación de la subrogación de personal en las empresas de seguridad: de una parte, entender las ventajas que reporta su aplicación; y de otra, averiguar si menoscaba el compromiso de los empleados que son subrogables. Para ello, tras realizar una revisión de la literatura científica del compromiso organizacional y del marco legal que regula este tipo de servicios, se presentan los resultados de una investigación empírica en una muestra de 706 trabajadores de una multinacional del sector. Posteriormente, se realiza el análisis de las ventajas que procura dicha figura jurídica, para concluir, finalmente, que se comporta como: un agente moderador de la complejidad asociada con la transmisión de empleados entre contratistas de seguridad; un elemento de estabilidad laboral que no tiene influencia en el compromiso del personal subrogable; y un factor que aporta certeza y calidad al servicio que reciben las empresas cliente de las de seguridad. Se derivan importantes implicaciones que resultan clarificadoras de las consecuencias que tendría para el sector que se limitara o impidiera su negociación entre patronal y sindicatos.

Palabras clave: Subrogación de Personal; Vigilantes de Seguridad; Subcontratación de Seguridad Privada; Compromiso con la Profesión.

Códigos JEL: M54, J41, J89, L24, D22.

\footnotetext{
Abstract:

${ }^{1}$ savila@eae.es, EAE Business School.

2 camlag@ceu.es, Universidad CEU San Pablo.

3 mpascualfaura@gmail.com, Universidad CEU San Pablo.

Recibido: 31 de marzo de 2020. Aceptado: 26 de noviembre de 2020.
}

Since the end of the last century, the private security sector in Spain has become an important economic and social agent, because of the impact of its turnover on the country's economy and the large number of stable jobs created by the legal concept of subrogation of personnel. The 
objective of this work is to answer two basic questions about subrogation of personnel in security companies: on the one hand, to understand the advantages of its application; and on the other hand, to find out if it undermines the commitment of the employees who are subrogated. To comply with this objective, after a review of the scientific literature on organizational commitment and the legal framework that regulates this type of service, the results of an empirical research on a sample of 706 workers from a multinational in the sector are presented. Subsequently, an analysis is made of the advantages provided by this legal figure, to conclude, finally, that it behaves as: a moderating agent of the complexity associated with the transfer of employees between security contractors; an element of labor stability that has no influence on the commitment of the subordinate personnel; and a factor that provides certainty and quality to the service received by the client companies from the security companies. There are important implications that clarify the consequences for the sector if negotiations between employers and unions were to be limited or prevented.

Key words: Subrogation of Personnel; Security guards; Private Security Sector; Commitment to the Profession

JEL Codes: M54, J41, J89, L24, D22.

\section{INTRODUCCIÓN}

Los sistemas relacionales que conforman la actividad humana (sociales, políticos, económicos) se transforman gradualmente, los tecnológicos no; estos últimos, cuando alcanzan una masa crítica de usuarios, se aceleran exponencialmente y provocan la fractura de los otros sistemas (Downes y Mui, 1999). De ahí que la tecnología se haya constituido en precursora de un cambio que deviene constante y que impacta notablemente en las relaciones que se establecen entre los trabajadores y sus organizaciones. Además, la situación de los empleados que trabajan en empresas de outsourcing -seguridad y limpieza entre otras- (Rivo, 1999) y de los que tienen contratos temporales y a tiempo parcial, es muy distinta de la que se daba en las décadas de los 60 y 70 del siglo pasado.

Derivado de lo anterior, resulta difícil que un empleado pueda desarrollar toda su vida laboral en una misma organización (Bauman, 2004). Como consecuencia, los sistemas de control burocrático de antaño que se basaban en la antigüedad y la experiencia (estar), han pasado a hacerlo en el desempeño (hacer), por lo que los empleados con mejores habilidades e información disponen de un poder negociador que no tenían sus predecesores (Cappelli, 2000).

Con base en dichas circunstancias, los trabajos de investigación sobre el compromiso de los empleados con la organización vienen siendo complementados por otros que estudian el compromiso de los trabajadores con su propia carrera, trabajo o profesión (Albrecht y Andreetta, 2011; Cohen, 2007; Cooper-Hakim y Viswesvaran, 2005; Garrido, 2016; Morrow, 2011). En este marco de referencia, muchos directivos se plantean qué cabe hacer para que no decaiga este tipo de compromiso por ser una actitud que favorece unas menores tasas de absentismo y rotación, y que se concreta precursora de un mejor desempeño y satisfacción de los clientes (Apascaritei y Elvira, 2018; Vecina y Chacón, 2013).

Por otra parte y en cuanto a las organizaciones empresariales, hay sectores, el de la seguridad privada entre ellos, en los que la existencia de la subrogación de personal hace posible que el empleado se sienta comprometido con su empresa en un entorno laboral estable. El sector de la seguridad privada en España, según los datos publicados por la Asociación Profesional de 
Compañías Privadas de Seguridad (APROSER, 2018), cuenta con 1.581 empresas, que facturan 4.032 millones de euros y dan empleo a 84.125 vigilantes de seguridad que son potencialmente subrogables.

Teniendo como fundamento lo apuntado, cabe preguntarse por: las ventajas que reporta para este sector la existencia de dicha figura jurídica; y el impacto que tiene en el compromiso de los trabajadores que son subrogables, puesto que su relación con la empresa se adivina más débil que la que pudieran tener los empleados que no lo son. Es por ello que el presente estudio sobre la subrogación de personal tiene un doble objetivo:

- Analizar las ventajas que se derivan de su desarrollo normativo y aplicación en el sector de la seguridad privada en España desde una triple perspectiva:

a) La propia de las empresas de seguridad privada.

b) La de los empleados de dichas organizaciones que poseen la condición de personal subrogable.

c) La de las empresas que son clientes de las de seguridad. personal.

- Y contrastar si el compromiso del personal subrogable es inferior al del resto del

Este trabajo está organizado de la siguiente manera. En la sección 2 se revisa la literatura científica más relevante sobre el compromiso organizacional. En la 3 se hace lo propio con el marco legal y jurídico que regula la actividad del sector. En la 4 se analizan las particularidades de este tipo de servicios. En la 5 se presenta el estudio empírico y sus resultados. En la 6 se discuten las ventajas de la subrogación misma bajo la triple perspectiva apuntada. Finalmente, en la sección 7, se recogen las principales conclusiones e implicaciones del estudio.

\section{COMPROMISO ORGANIZACIONAL}

El estudio del Compromiso Organizacional se inicia a mediados del siglo pasado, teniendo como uno de sus primeros referentes al modelo propuesto por Becker (1960); su perspectiva, conocida como compromiso de cálculo o intencionado, entiende que existe compromiso "cuando una persona, por inversiones pretéritas, vincula intereses ajenos con una constante línea de actuación” (p.32). El empleado sopesa continuar en la organización en función del coste de oportunidad que le pudiera suponer un cambio de empresa.

Más tarde, surge la perspectiva actitudinal del compromiso, que lo define como una ligazón psicológica de carácter afectivo entre el empleado y su organización que le empuja a actuar por encima de su obligación contractual (Mowday et al., 1974). En el año 1979, el equipo de Porter desarrolla y valida el cuestionario denominado OCQ (Organizational Commitment Questionnaire) (Mowday et al., 1979), herramienta que se sigue empleando con frecuencia en estudios sobre este tipo de compromiso (Ávila, 2019; Ávila y Pascual, 2019; Bermejo et al., 2014; González et al., 2014).

Posteriormente, Meyer y Allen proponen un modelo compuesto por dos dimensiones: afectiva de carácter actitudinal y relacionada con la visión de Porter, y de continuidad en base al compromiso de cálculo o intencionado de Becker, que se concretaba a modo de síntesis de las dos perspectivas que predominaban hasta esos momentos (Meyer y Allen, 1984). Su propuesta se completa más tarde con la inclusión de la dimensión normativa, que se asocia con la obligación moral que siente el trabajador por alcanzar los objetivos de la organización (Allen y Meyer, 1990). Quedando así constituido el modelo tridimensional de compromiso que más base investigadora ha acumulado (Kam et al., 2016; Neves et al., 2018).

No obstante, en la actualidad existe un fuerte debate sobre lo adecuado del modelo, puesto que según algunos autores la dimensión de continuidad explicaría la causa por la que un 
empleado quiere continuar en la organización pero no informa de su empeño por alcanzar el logro colectivo (Ashman y Winstanley, 2006; Jong-Wook et al., 1997). Como refiere Swailes (2002), resulta difícil que los directivos de una empresa puedan considerar comprometidos a aquellos trabajadores que continúan en ella por falta de alternativas; de ahí que, abundando en el razonamiento anterior y según Ávila y Pascual (2020), no se pueda calificar de comprometido al trabajador que solo persigue la defensa de sus intereses.

También, y derivado de la considerable superposición que existe entre las componentes afectiva y normativa, se discute la utilidad de mantenerlas diferenciadas (Jong-Wook et al., 1997). Cohen (2003) se suma a la crítica, si las escalas no son diferentes para qué mantenerlas, crítica que continúa con Bergman (2006), "sin demostrar efectos diferenciales en los resultados, la utilidad de retener ambos constructos no está clara” (p.659). No obstante y al margen de cuestionar la utilidad de mantener las dos escalas, surge la crítica apuntada por Ávila y Pascual (2020) de que no se debe confundir la ligazón afectiva que tiene el trabajador con un proyecto y empresa concreta (dimensión afectiva) con un compromiso del trabajador con su propia ética personal (dimensión normativa); ética, que estará siempre presente en él al margen de la organización en la que este se encuentre.

Derivado de lo anterior, cada vez hay más trabajos que estudian el compromiso organizacional desde una visión unidimensional y afectiva, por lo que a entender de Mercurio (2015), la dimensión afectiva se debiera considerar la esencia de lo que es este tipo de compromiso, y en base a ella se debiera orientar su estudio futuro (Cernas y Nava, 2019).

El presente trabajo se ha desarrollado en base a considerar que el compromiso es "una ligazón afectiva que une al empleado con la organización, que le impulsa a actuar con unos altos niveles de esfuerzo y dedicación en el logro de las metas y objetivos de la empresa" (Ávila y Pascual, 2020, p.216). Esto es, se tiene como un constructo unidimensional de carácter afectivo.

\section{VERTIENTE NORMATIVA Y LEGAL DEL SECTOR DE LA SEGURIDAD PRIVADA EN ESPAÑA}

El marco legal del sector de la seguridad privada en España encuentra fundamento en los siguientes referentes:

- La ley de Seguridad Privada, con una normativa específica para el sector.

- Las leyes y normas de carácter laboral que dan amparo legal a la figura de la subrogación de personal recogida en el convenio estatal de empresas de seguridad, que son comunes a otras actividades como las de limpieza viaria, limpieza de oficinas, jardinería, personal de servicios en tierra de los aeropuertos...

\subsection{Ley de Seguridad Privada}

En la actualidad, el sector de la seguridad privada en España se encuentra regulado por la Ley 5/2014, de 4 de abril, de Seguridad Privada; teniendo los fines recogidos en los apartados a, b y c, del Capítulo I, artículo 4, siguientes:

- Satisfacer las necesidades de seguridad e información de los usuarios de dichos servicios.

- Procurar la inmunidad y privacidad de las personas y bienes cuya seguridad se les encomiende frente a amenazas deliberadas y riesgos accidentales.

- Contribuir a garantizar la seguridad pública como complemento al monopolio de la seguridad que corresponde al Estado. 
Dicha ley, en el artículo 5 del mismo capítulo, regula, entre otras, las actividades de:

- La vigilancia y protección de bienes en instalaciones y eventos públicos y privados, así como de las personas que allí se encuentren.

- La protección de personas que tengan la condición legal de autoridad.

Por lo que toda empresa que desarrolle actividades como las anteriormente citadas, debiendo ser de seguridad y estar inscrita en el Ministerio del Interior, vendrá obligada por la ley de Seguridad Privada.

\subsection{Leyes y normas de carácter laboral. Bases jurídicas de la capacidad reguladora de la subrogación de personal por parte de las Organizaciones Sindicales y Empresariales}

Las empresas de seguridad, desarrollan un tipo de servicio que requiere la presencia de vigilantes y responsables de equipo en las instalaciones de sus clientes. Dichos clientes, al aceptar ofertas más ventajosas y cambiar de empresa proveedora de este tipo de servicio, propician que el personal que tienen asignado, por efecto de la subrogación, cambie de empresa y de uniforme, continuando con un cometido y lugar de trabajo en el que ya operaban con anterioridad.

La necesidad de dotar de estabilidad al empleo, junto con otras circunstancias que se tratarán en el apartado 6, son las que han propiciado la fijación de determinadas reglas y garantías que persiguen beneficiar al trabajador y a la empresa. Corresponde a las Organizaciones Sindicales y Empresariales, mediante inclusión en el Convenio Colectivo correspondiente, regular las cláusulas de subrogación de personal que procuran estabilidad al empleado y certeza a las empresas.

Varias son las fuentes de fundamento jurídico que dan soporte legal a la capacidad reguladora de las Organizaciones Sindicales y de la Patronal del sector en base a la subrogación de personal. Desde lo genérico a lo particular, se mencionan las siguientes:

- Constitucionales

- Legales

- De la Negociación Colectiva

- Del Convenio Colectivo Estatal para Empresas de Seguridad

\subsubsection{Bases Constitucionales}

Los artículos 7 y 37 de la Constitución, dan amparo a quienes se les reconoce la capacidad de negociar y acordar las condiciones que, como la subrogación de personal, se deben recoger en convenio. El primero de ellos establece que corresponde a los sindicatos de trabajadores y a las asociaciones empresariales la defensa de sus intereses económicos y sociales. El segundo reconoce la fuerza vinculante de los convenios que acuerden, y el derecho que les asiste para la negociación colectiva y para la adopción de medidas de conflicto colectivo.

\subsubsection{Bases Legales}

Encuentran fundamento en el Estatuto de los Trabajadores; en particular, la referida al artículo 44.1 sobre la sucesión de empresa, que señala: “...quedando el nuevo empresario subrogado en los derechos y obligaciones laborales y de Seguridad Social del anterior...”, por lo que la nueva adjudicataria se verá obligada a aceptar las condiciones que tenían los empleados que estaban adscritos al servicio ( salario, pluses, antigüedad...). 
Más adelante, el artículo 85.1, establece: “Dentro del respeto a las leyes, los convenios colectivos podrán regular materias de índole económica, laboral, sindical y, en general, las que afecten a las condiciones de empleo...” apunte que daría soporte a la subrogación de personal.

\subsubsection{Bases de la Negociación Colectiva}

Son bases que encuentran soporte en lo que ha venido en llamarse el diálogo social; diálogo que, en forma de Acuerdos para la Negociación Colectiva (ANC), se han suscrito desde finales del siglo pasado (1997) entre los sindicatos CC.OO - UGT y las organizaciones empresariales CEOE - CEPYME. En todos ellos y de forma recurrente, se menciona lo acordado en el Capítulo II del AENC (Acuerdos para el Empleo y la Negociación Colectiva) 2015-2017 (BOE núm.147, sábado 20 de junio de 2015. Sec. III.): “...El mantenimiento y recuperación del empleo. El fomento de la estabilidad del empleo...como garantía de competencia para las empresas y de seguridad para los trabajadores” (p.51604). Más tarde (BOE núm. 173, miércoles 18 de julio de 2018), se alcanzó, entre otros, un acuerdo para mantener el empleo sin que este perjudique la retribución y protección social del trabajador.

En definitiva, tanto La Constitución como el Estatuto de los Trabajadores y los AENC, se constituyen en fundamento y referencia de la buscada estabilidad laboral y del Convenio Colectivo Estatal para empresas de Seguridad en España.

\subsubsection{El Convenio Colectivo Estatal para Empresas de Seguridad. 2017-2020.}

Dada la naturaleza, circunstancias y características del sector, y atendiendo a garantizar la estabilidad en el empleo -no de un concreto puesto de trabajo-, el Convenio Colectivo recoge un articulado que trata de forma específica la figura de la subrogación. Señalando, en su artículo 14 (capítulo III):

La subrogación se produce cuando una empresa sustituye de forma total o parcial a otra en la prestación de los servicios contratados por un cliente, público o privado, cualquiera que fuera la causa, en los supuestos y términos establecidos en este convenio.

Subrogación, que obliga a la nueva adjudicataria del servicio "a integrar en su plantilla, subrogándose en sus contratos de trabajo, a los trabajadores de la empresa cesante en el servicio... siempre que se acredite el requisito de antigüedad establecido...”, que queda fijado en los siete meses que preceden de forma inmediata a la fecha en que se produzca la subrogación.

No obstante, a partir de septiembre de 2018, se ha producido un cambio de doctrina en la sucesión de empresas (Sentencia del Tribunal Supremo de 27 de septiembre de 2018 -R ${ }^{\circ}$ 2747/2016-) que fija que no se pueden limitar por convenio los efectos que pudiera tener la subrogación en los trabajadores; esto es, el nuevo adjudicatario del servicio quedará obligado solidariamente a las deudas laborales y de seguridad social del anterior empleador. Por lo que el escenario ha mutado, de una nueva adjudicataria que no tenía responsabilidades con las deudas que hubiere contraído el anterior empleador con los trabajadores subrogados, a otro en el que sí las tiene.

\section{PARTICULARIDADES DE LOS SERVICIOS DE VIGILANCIA EN LA SEGURIDAD PRIVADA}

Varias son las características que, comunes con otro tipo de servicios intensivos en mano de obra, se presentan en el sector de la seguridad privada tanto en la contratación como en la explotación y su finalización. 
En cuanto a su contratación, en muchas ocasiones los servicios ofertados se materializan como la expresión de una necesidad de carácter público, que presenta una clara incidencia en lo más básico y cotidiano de la actividad ciudadana: su seguridad (ADIF, RENFE, aeropuertos, centrales nucleares, edificios públicos...). El sector público representa un 20 por cien del total del mercado de la seguridad privada (APROSER, 2018). En otras muchas más se concreta como la manifestación de una necesidad de la empresa privada (80 por cien del mercado), que requiere de seguridad para el normal desarrollo de su actividad. En ocasiones, cuando la adjudicación de un servicio se sujeta a una limitación temporal, la necesidad que lo ampara deviene en permanente.

En la explotación, los servicios de seguridad son generadores de contratación intensiva en mano de obra. El número de vigilantes de seguridad privada en activo es de 84.125 (APROSER, 2018). Y en su finalización, resulta habitual que al término de un servicio que se licitó inicialmente como una única contrata, se divida en varios lotes y servicios para licitaciones posteriores; lo contrario también sucede (hacer un solo lote de varios). La sucesión de contratas se presenta como una dinámica de lo más habitual en el sector.

\subsection{La subrogación de personal y el sector de la seguridad privada.}

Las empresas del sector de la seguridad privada en España están obligadas por convenio a la subrogación del personal que trabaja en las instalaciones de sus clientes (artículo 14, capítulo III). Organizaciones en las que conviven dos tipos de realidades diferentes que son complementarias entre si:

- La del personal subrogable. Empleados que trabajan en las instalaciones de las empresas clientes de la suya de seguridad, que permanecen en dichas instalaciones integrándose en la plantilla de la nueva adjudicataria del servicio cuando cambian de proveedor. En este caso se hace referencia a las categorías sociolaborales de: vigilantes de seguridad y responsables de equipo.

- La del personal de estructura. Trabajadores que realizan su cometido en las instalaciones de la propia empresa de seguridad y que no son objeto de subrogación. Empleados que al no ser subrogables dan estabilidad organizativa a la empresa. En este caso se hace referencia a las categorías socio-laborales de: personal de base sin responsabilidad sobre terceros, supervisores, y directivos.

A continuación, se muestra el impacto que tiene el sector de la seguridad privada en la economía y en el empleo del país (Tabla 1):

\section{TABLA 1: DATOS SECTOR DE LA SEGURIDAD PRIVADA}

\begin{tabular}{ccccc}
\hline $\begin{array}{c}\text { Número de } \\
\text { Empresas }\end{array}$ & $\begin{array}{c}\text { Facturación del } \\
\text { Sector de Seguridad } \\
\text { Privada }\end{array}$ & $\begin{array}{c}\text { Procedencia de la } \\
\text { Facturación }\end{array}$ & $\begin{array}{c}\text { Personal } \\
\text { Subrogable }\end{array}$ & $\begin{array}{c}\text { Contrato } \\
\text { Indefinido }\end{array}$ \\
Seguridad & $\begin{array}{c}\text { Prillones de euros) } \\
1.581\end{array}$ & $\begin{array}{c}\text { Privado } \\
\text { Público }\end{array}$ & Vigilantes & Vigilantes \\
\hline
\end{tabular}

Fuente: (APROSER, 2018, p.1).

En la Tabla 1 se aprecia el número de empresas y empleados de seguridad que son potenciales beneficiarios de las ventajas que supone tener recogida en convenio la figura de la subrogación de personal. El tejido empresarial del sector viene formado por una mayoría de empresas (84,7 por cien) con plantillas menores de 50 trabajadores, y una minoría (0,3 por cien) que cuenta con 5.000 o más empleados; minoría de la que solo la empresa Prosegur cotiza 
en Bolsa en España (Yoldi, 2019). La tecnología ha irrumpido con fuerza en el sector de forma que la protección del valor (personas y bienes) pasa por el uso prioritario de esta, que se concreta, junto con las personas y los procedimientos, en el tercer pilar de la seguridad privada (Eulen, 2019).

\subsection{Las empresas que son clientes de las empresas de seguridad.}

Desde una perspectiva empresarial distinta, las empresas que son clientes de las organizaciones de seguridad y que reciben de estas sus servicios, están contratando un tipo de actividades que se denominan de apoyo en su cadena de valor (en la Figura 1. Actividades de infraestructura. Servicios generales), porque cumplen con la función de dar soporte a las actividades de producción o de servicio que le son propias y que se denominan primarias (Porter, 1989).

\section{FIGURA 1: CADENA DE VALOR DE LAS EMPRESAS CLIENTE DE LAS ORGANIZACIONES DE SEGURIDAD}

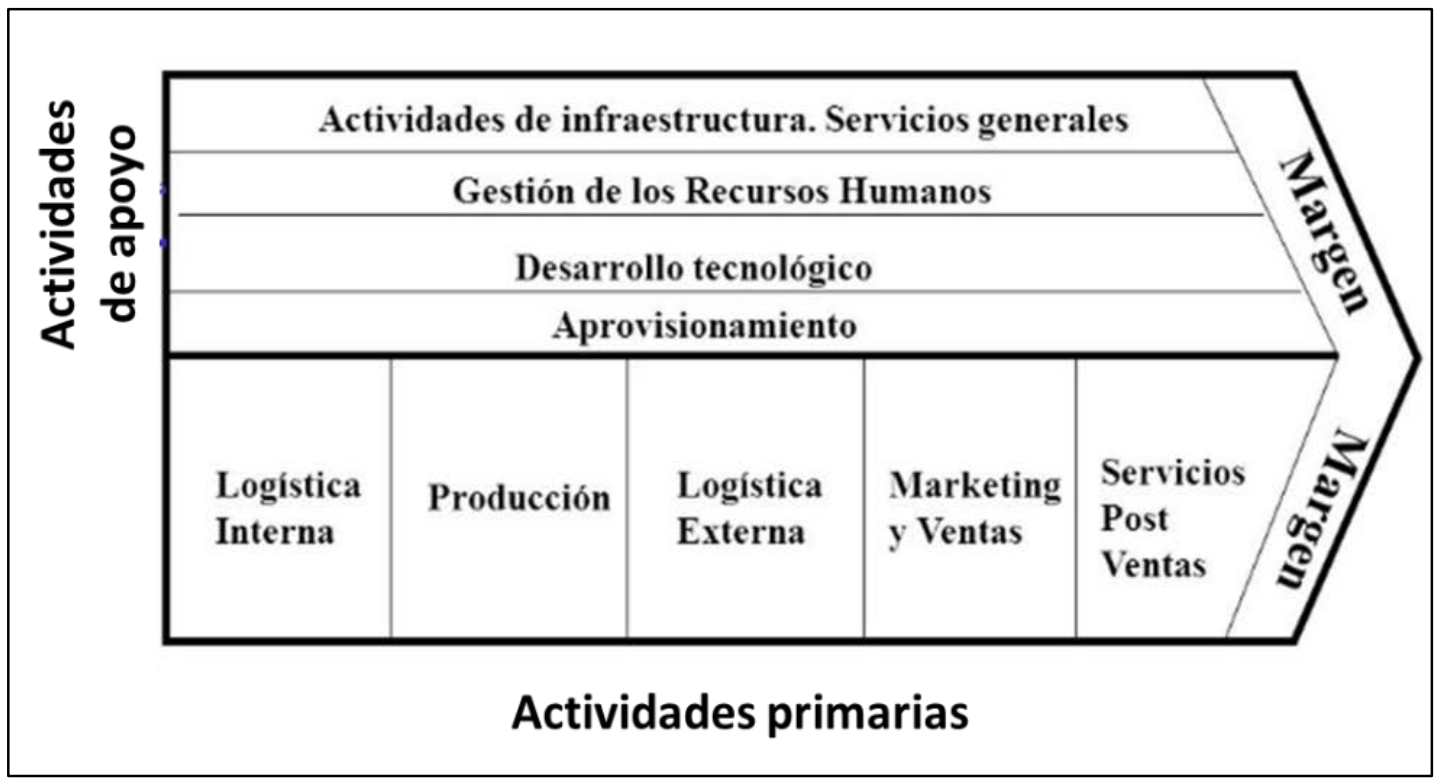

Fuente: (Porter, 1989, p.55).

La empresa de seguridad, a pesar de que realiza actividades de apoyo y agrega valor a su empresa cliente -itinerario 2 de la figura 2-, no incide en el valor agregado real que perciben los compradores de sus productos o servicios -itinerario 1 de la figura 2- (Haaz, 2010). De ahí que los servicios de seguridad, al formar parte habitual del itinerario 2, se presenten como secundarios de la actividad principal de sus clientes y se valoren como indiferenciados y de menor importancia. 


\section{FIGURA 2: CADENA DE VALOR Y SERVICIOS DE SEGURIDAD}

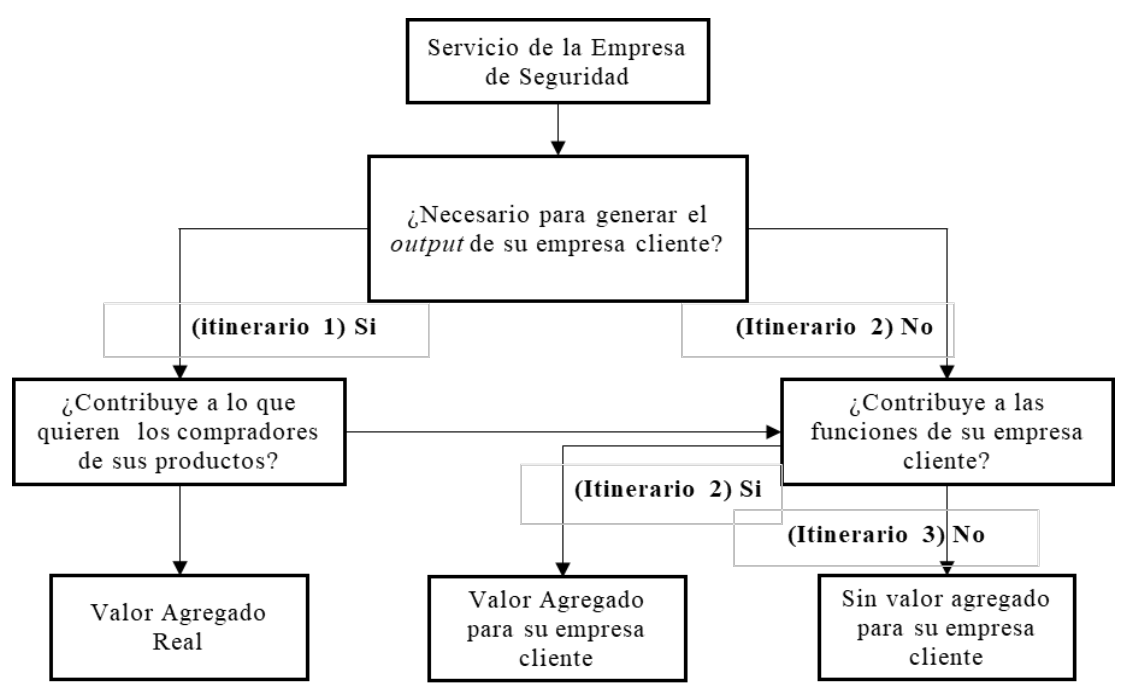

Fuente: (Haaz, 2010).

\section{ESTUDIO EMPÍRICO SOBRE EL COMPROMISO EN UNA MULTINACIONAL DEL SECTOR DE SEGURIDAD PRIVADA EN ESPAÑA}

A continuación, se muestran algunos resultados de la investigación empírica realizada en una multinacional del sector de la seguridad privada en España, en la que entre otras hipótesis se contrasta si existen diferencias de compromiso entre el personal subrogable y el de estructura.

\subsection{Participantes}

El estudio fue realizado en base a los datos recabados en la multinacional referida durante el mes de mayo de 2017. La muestra quedó constituida por 706 trabajadores que estaban distribuidos por toda la geografía española y tenían una edad media de 42 años, siendo la máxima de 64 años y la mínima de 20 años. La distribución por sexos fue de 580 hombres (82,2 por cien) y 126 mujeres (17,8 por cien). En la Tabla 2 se muestra la ficha técnica de la investigación.

\section{TABLA 2: FICHA TÉCNICA DE LA INVESTIGACIÓN}

\begin{tabular}{ll} 
& Trabajadores de la Multinacional \\
Universo & SECURITAS en España \\
Ámbito geográfico & Nacional \\
Población & 10.230 empleados \\
Tamaño muestra real & 706 empleados \\
Ratio de Respuesta & $6,90 \%$ \\
Diseño muestral & Encuesta por internet \\
Error muestral & $4,68 \%$ \\
Intervalo de confianza & $99 \%(p=q=0,5)$ \\
Período de recogida de información & Mayo de 2017 \\
Información recogida mediante cuestionario & 7 ítems \\
\hline
\end{tabular}

Fuente: Elaboración propia. 
En relación con el desglose de personal por categorías sociolaborales y en atención a su condición de ser o no ser personal subrogable, se muestra la Tabla 3.

\section{TABLA 3: DISTRIBUCIÓN DE CATEGORÍAS SOCIOLABORALES. PERSONAL DE ESTRUCTURA Y SUBROGABLE}

\begin{tabular}{crcc}
\hline & & $\begin{array}{c}\text { Personal de } \\
\text { Estructura }\end{array}$ & $\begin{array}{c}\text { Personal } \\
\text { Subrogable }\end{array}$ \\
\hline Directivos & Directivos & 37 & \\
\hline \multirow{2}{*}{ Mandos Intermedios } & Supervisores & 20 & \\
& Responsables de Equipo & & 62 \\
\hline \multirow{2}{*}{ Personal de base } & Comercial y Administrativo & 46 & 541 \\
\cline { 2 - 4 } & Vigilantes de Seguridad & & 603 \\
\hline
\end{tabular}

Fuente: Elaboración propia.

Tal como se puede observar en la Tabla 3, hay dos tipos de mandos intermedios: supervisores, que forman parte de la estructura de la empresa y que por tanto no son subrogables, y responsables de equipo, que dependen de ellos y sí que lo son. Lo propio ocurre con el personal de base, constituido en la estructura por personal sin responsabilidad sobre terceros que realiza cometidos de índole comercial o administrativa, y en el personal subrogable por los vigilantes de seguridad.

\subsection{Instrumento}

La herramienta empleada para la medida del compromiso organizacional fue una versión traducida al español del OCQ -Organizational Commitment Questionnaire- de Porter (Mowday et al., 1979, p. 228); cuestionario en el que se adaptaron los ítems de expresión negativa a otros que mantenían su misma intención y se presentaban afirmativos. En la actualidad, el cuestionario de Porter es una herramienta ampliamente utilizada en este tipo de investigaciones (Bermejo et al., 2014; González et al., 2014; González et al., 2014; S. Lee y Gao, 2005; Swailes, 2002). Las respuestas al cuestionario debían ser dadas en una escala Likert de 5 alternativas, variando sus apreciaciones de respuesta entre: (1) "Desacuerdo Total" a (5) "Acuerdo Total".

Para su elaboración se emplearon técnicas de análisis factorial exploratorio y confirmatorio que se realizaron con los programas SPSS_24 y Amos 25 respectivamente. En primer lugar, se realizaron varios análisis factoriales exploratorios (rotación oblicua tipo Oblimin), que precedieron a un análisis factorial de segundo orden, que sirvió a su vez como base de propuesta para un análisis factorial confirmatorio. Derivado de dichos análisis se obtuvo un cuestionario final que está formado por siete ítems en su dimensión afectiva del compromiso -alfa de Cronbach 0,92- (Ávila y Pascual, 2019, p. 179). (Anexo 1)

Los ítems que constituyen dicho cuestionario y dimensión se refieren a las siguientes tres perspectivas: de logro, sobre la actitud proactiva que muestra el trabajador por conseguir los objetivos de la empresa (ítems 3, 4 y 6); evocada, sobre la valoración que hace el empleado de su experiencia laboral (ítems 2, 5 y 7); de branding, sobre la imagen de marca que proyectan los trabajadores de su organización (ítem 1). 


\subsection{Análisis multivariante y algunos resultados}

Posteriormente, se realizó, entre otros análisis estadísticos, un análisis multivariante (prueba ANOVA con empleo del programa SPSS_24) con la finalidad de analizar si existen diferencias de compromiso entre el personal subrogable y el de estructura; contrastando, entre otras, las siguientes hipótesis: $\mathrm{H}$ 1. El nivel de compromiso del grupo "responsables de equipo" es igual al del grupo "directivos"; y $\mathrm{H} \mathrm{2}$. El nivel de compromiso del grupo "vigilantes de seguridad” es igual al del grupo "comercial y administrativo". Análisis que puso de manifiesto el cumplimiento de las dos hipótesis planteadas (Tabla 4).

\section{TABLA 4:CONTRASTE DE DIFERENCIA DE MEDIAS DEL COMPROMISO ORGANIZACIONAL (PRUEBA ANOVA)}

\begin{tabular}{clcccc}
\hline Grupos & Categorías & Muestra & por cien & Media & $\begin{array}{c}\text { Mann-Whitney } \\
\text { (p-value) }\end{array}$ \\
\hline G1 & Directivos & 37 & 5,39 & 4,27 & G1-G2 (0,237) \\
G2 & Comercial y Administrativo & 46 & 6,71 & 4,06 & G1-G3 (0,744) \\
G3 & Responsables de Equipo & 62 & 9,04 & 4,24 & G1-G4 (0,010) \\
G4 & Vigilantes de Seguridad & 541 & 78,86 & 3,82 & G2-G3 (0,369) \\
& & & & & G2-G4 (0,137) \\
& & & & & G3-G4 (0,004) \\
\hline
\end{tabular}

Fuente: Elaboración propia.

En la tabla 4 se pueden apreciar los contrastes de dichas hipótesis a partir de la prueba ANOVA realizada; así, en H1, cuando se compara el nivel de compromiso del grupo "responsables de equipo" (G3:4,24) y del grupo “directivos” (G1:4,27), se obtiene un p-value de 0,744 , lo que indica que no existen diferencias significativas entre el nivel de compromiso de ambos colectivos; esto es, el nivel de compromiso del personal subrogable que se emplea en labores de mando intermedio (responsable de equipo) y del personal directivo que no es subrogable resulta similar.

En H2, cuando se hace lo propio con el grupo “vigilantes de seguridad” $(\mathrm{G} 4: 3,823)$ y el grupo “comercial y administrativo" (G2:4,062), se obtiene un p-value de 0,137 , lo que indica que no se aprecian diferencias significativas entre el compromiso de ambos grupos. La falta de responsabilidad sobre terceros iguala en su nivel de compromiso al personal que es subrogable $\mathrm{y}$ al de estructura.

Con lo que se rechaza la hipótesis de que el personal subrogable es menos comprometido que el personal de estructura. Estos resultados están en línea con la investigación realizada por Ávila (2019), en la que se refiere: "El criterio que delimita la expresión de un mayor nivel de compromiso organizacional lo fija la posibilidad de tener o no tener responsabilidad sobre terceros y no la de ser o no ser personal subrogable” (p. 368).

De ahí que se pueda afirmar que la existencia de la figura jurídica de la subrogación de personal no se muestra inductora de un menor nivel de compromiso en los empleados que son potencialmente subrogables. 


\section{ANÁLISIS DE LAS VENTAJAS QUE SE DERIVAN DE LA APLICACIÓN DE LA FIGURA JURÍDICA DE LA SUBROGACIÓN DE PERSONAL}

La subrogación de personal es un elemento que proporciona estabilidad laboral al empleado y certeza a las empresas; además, ofrece ventajas que se derivan del compromiso que manifiesta el personal subrogable con su profesión y que benefician a la organización en su conjunto. Compromiso, que en lógica consecuencia también favorece a las empresas que son clientes de las de seguridad. A continuación, se exponen dichas ventajas.

\subsection{Ventajas para la empresa de servicios de seguridad}

Disponer en convenio de una figura legal como la subrogación de personal, se materializa como un elemento de orden al regular las habituales sucesiones de empresas que se dan en el sector de la seguridad, que procura ventajas como las que se señalan a continuación:

- Facilita los frecuentes cambios de contratas que tienen lugar en la actividad. La complejidad que se deriva de las transmisiones de empleados entre contratas, queda moderada por una figura jurídica que aporta claridad y transparencia al proceso a la vez que evita su excesiva judicialización (Moreno-Jiménez y Díaz Gracia, 2018).

- Garantiza la continuidad operativa de los servicios. La nueva adjudicataria, al continuar con la plantilla que operaba con anterioridad en las instalaciones del cliente, se beneficia con la incorporación de trabajadores que conocen el servicio y que en el 80 por cien de los casos gozan de contrato indefinido (APROSER, 2018). De no ser posible la subrogación de empleados, cada nueva adjudicación se concretaría con los problemas propios de un inicio constante de actividad.

- Incrementa la productividad y competitividad del sector. La estabilidad en el empleo, en la medida que evita la rotación de personal, contribuye a que la productividad y competitividad de las empresas de seguridad mejoren al no interrumpir la curva de aprendizaje (Olea et al., 2018).

- Evita que las empresas tengan que asumir un constante goteo de indemnizaciones. A la finalización de cada contrata, se produciría una gran cantidad de despidos que traerían como consecuencia un elevado coste indemnizatorio, lo que a su vez se traduciría en un importante desequilibrio económico-financiero, que vendría derivado del elevado porcentaje (80 por cien) que suponen los costes de personal en las cuentas de resultados de las empresas del sector. A modo de ejemplo, sobre unas ventas totales de 92.196,8 millones de $\mathrm{Kr}^{4}$ de la multinacional de seguridad SECURITAS, los gastos de producción (fundamentalmente de personal operativo) se situaron en 75.196,8 millones de $\mathrm{Kr}$, lo que supone un 81,56 por cien de la cifra de ingresos (SECURITAS, 2017, p.46).

- Propicia la paz social. La subrogación, al procurar una menor conflictividad laboral, se concreta como un elemento evocador de seguridad jurídica y empleabilidad (UGT, 2020).

Asimismo, mantiene los niveles de:

- Calidad del servicio. La subrogación, al dar continuidad a la plantilla e impedir que se pierda su conocimiento y experiencia, se plasma como un elemento estabilizador de la calidad del servicio (Madrigal y David, 2015).

${ }^{4} \mathrm{Kr}=$ Corona Sueca 
- Incidencia de riesgos laborales. Por la misma razón que en el punto anterior, se conservan o incluso mejoran los índices de incidencias laborales. La subrogación, al potenciar el papel moderador de los contratos indefinidos (el 80 por cien ya comentado), procura unos menores índices de siniestralidad laboral, “...los contratos temporales tienen un porcentaje superior de incidencia laboral. El índice de incidencia de los trabajadores (asalariados) temporales fue 1,8 veces mayor que el de los trabajadores indefinidos” (Moreno-Jiménez y Díaz Gracia, 2018, p.139).

- No influye en el nivel de compromiso de los empleados potencialmente subrogables. La subrogación no se concreta como un factor inductor de un menor nivel de compromiso organizacional, ya que como se ha expuesto en el apartado 5, se relaciona positivamente con tener responsabilidad sobre terceros y no con el hecho de ser personal subrogable. Así, se pudo contrastar en la investigación realizada que los vigilantes de seguridad muestran un nivel de compromiso similar al del personal de base en la estructura, de la misma forma que ocurre con los responsables de equipo y los directivos.

\subsection{Ventajas para los empleados subrogables}

Las ventajas que proporciona la figura de la subrogación no quedan agotadas con lo expresado en el apartado anterior, también, y en el plano laboral del empleado se dan las que se exponen a continuación:

- Reduce la temporalidad en el empleo. La subrogación, al procurar una menor temporalidad en beneficio de una mayor continuidad laboral, se manifiesta como una fuente de empleo “fijo” y de calidad; “...el 80 por cien disfruta de una modalidad de contratación de carácter indefinido, síntoma de la histórica apuesta del sector por la calidad y estabilidad en el empleo" (APROSER, 2018, p.1)

- Propicia la estabilidad laboral. Puesto que dota de seguridad y certeza tanto a los trabajadores como a la empresa en todos aquellos escenarios en los que la sucesión de contratas es frecuente. Tener unas reglas claras permite que se concilien los intereses de estabilidad de los trabajadores y de las organizaciones para las que estos trabajan (Hernández, 2019).

- Favorece la capacitación profesional de los empleados. Ya que se materializa como un instrumento de capacitación profesional a la vez que se plasma como un agente activo de la prevención de riesgos laborales. Al dar continuidad al trabajador se evita que todo cambio de proveedor se constituya en un cambio constante de plantilla y se facilita el crecimiento de su pericia profesional (La Vanguardia, 2019).

- Garantiza una menor conflictividad laboral. Al procurar una paz social que resulta muy necesaria para el normal desarrollo de la actividad en aeropuertos, centros de comunicación, centrales nucleares...(EUROPA PRESS, 2019), y para la vida laboral del empleado que puede transitar de una empresa a otra sin tener que recurrir a la vía judicial en defensa de sus intereses.

\subsection{Ventajas para las empresas que son clientes de las empresas de seguridad}

Las ventajas que disfrutan las empresas clientes de las de seguridad se derivan de las que tienen las empresas mismas de seguridad y sus empleados, ya que la subrogación les garantiza:

- Proveerse de una estructura de costes variables. Al reducir los costes fijos e incrementar la predictibilidad de los variables -los costes variables son fijos 
unitariamente (Pereira et al., 1989)- se reduce el apalancamiento operativo de la propia empresa y su riesgo económico asociado (Rivo, 1999).

- La reducción de riesgos que se derivan de los cambios tecnológicos. Al trasladar a sus proveedores los riesgos derivados de la obsolescencia tecnológica que pudiera darse en su operativa.

- Continuidad en la operativa de los servicios que no le son propios pero que forman parte de su cadena de valor. Las empresas de seguridad agregan valor a las organizaciones que son clientes de sus servicios y les procuran el normal desarrollo de sus actividades. Servicios que incorporados a su cadena de valor, se constituyen en actividades de apoyo de las primarias que les son propias (Porter, 1989).

- Paz social. La figura de la subrogación, al garantizar la continuidad, retribución y antigüedad de los empleados a efectos retributivos, procura la ausencia de conflictividad en las transiciones entre empresas del sector y asegura el normal desarrollo de las operaciones en las organizaciones en las que se desempeña el personal subrogable.

- Que los índices de incidencia relativos a los riesgos laborales se mantienen o incluso mejoran. Por lo que se facilita la prevención, el seguimiento y cumplimiento por parte de sus proveedores de seguridad de la normativa de riesgos laborales, ya que el apartado 3 del artículo 24 de la Ley de Prevención de Riesgos Laborales hace responsable al contratista principal (la empresa cliente de la de seguridad) del cumplimiento de esta:

Las empresas que contraten o subcontraten con otras la realización de obras o servicios correspondientes a la propia actividad de aquellas y que se desarrollen en sus propios centros de trabajo, deberán vigilar el cumplimiento por dichos contratistas y subcontratistas de la normativa de prevención de riesgos laborales.

- Un servicio de seguridad cada vez más productivo y eficiente. Lo que pudiera permitir la captura de sinergias y economías de escala en el desarrollo del servicio, derivándose la consecuente reducción de costes. En ocasiones, la acumulación de experiencia y conocimiento del servicio propicia una nueva operativa que es inductora de un menor coste asociado.

- Que el personal subrogable presente el mismo grado de compromiso que el de estructura con el mismo nivel de responsabilidad. Circunstancia que se deriva de la investigación referida en el apartado 5 , y de la que cabe deducir que el nivel de compromiso de los trabajadores subrogables es el mismo que el que tendrían si formaran parte de la estructura de la empresa cliente.

\subsection{Desventajas}

La principal desventaja de la subrogación de personal se deriva de la estabilidad laboral que propicia; así, la acumulación de antigüedad del personal subrogable, y el consecuente incremento de su coste indemnizatorio, se materializa como un elemento que disuade de la desvinculación del personal falto de rendimiento. En ocasiones, su coste supera o disminuye notablemente el margen de contribución del contrato, de ahí que el nuevo contratista, ante la disyuntiva de desvincular o no a un empleado, opte por continuar con la dinámica del servicio.

\section{CONCLUSIONES}

Desde finales del siglo XX, las denominadas nuevas tecnologías desafian constantemente los equilibrios que se dan entre las organizaciones y sus trabajadores; también, y gracias a la 
generalización del trabajo temporal y a tiempo parcial y del outsourcing, se ha producido un cambio en la forma en que se contempla la relación del empleado con su empresa. Derivado de lo anterior y ligado al fenómeno del outsourcing, se ha desarrollado un marco legal de referencia que trata de ordenar y facilitar, mediante la subrogación de personal, la transición de personal entre empresas proveedoras de servicios. Servicios que como los de seguridad privada, tienen una gran importancia para el normal desarrollo de la actividad económica y social del país. Ante esta situación, cabe preguntarse por las ventajas que supone la aplicación de dicha figura jurídica y el impacto que tiene en el compromiso organizacional de los trabajadores que pueden ser subrogados.

El objetivo de este trabajo ha sido discutir las ventajas que reporta a las empresas y a sus empleados y clientes, la aplicación de la subrogación de personal en el sector de la seguridad privada en España, y contrastar el impacto que tiene en el compromiso organizacional de los trabajadores que son subrogables.

Derivado del análisis de la literatura científica más relevante sobre el compromiso organizacional, se realizó la parte empírica de esta investigación en base a considerar que el compromiso es una ligazón afectiva de carácter unidimensional.

Respecto al marco legal y jurídico de la subrogación de personal, concluir que se muestra lo suficientemente desarrollado y contrastado para poder afirmar que sí que favorece la subrogación del personal y el normal desarrollo de la actividad.

También, y a través del estudio empírico realizado, se ha mostrado evidencia de que no se aprecian diferencias de compromiso entre el personal subrogable y el personal de estructura, por lo que cabe concluir que el compromiso del personal subrogable no se muestra afectado por la aplicación de dicha figura jurídica.

En cuanto al análisis de las ventajas que reporta a las empresas de seguridad, se concluye que la subrogación de personal favorece el normal desarrollo de los cambios que se producen entre contratas del sector, lo que propicia la continuidad y paz social en el servicio y garantiza que sea más eficiente y productivo. Al no interumpir la curva de aprendizaje procura unos mejores índices de calidad y de prevención de riesgos laborales.

Por otra parte, los empleados que son subrogables se ven favorecidos en su capacitación profesional y estabilidad laboral, a la vez que se benefician de certeza judicial en las transiciones que se dan entre empresas contratistas de seguridad.

En relación a las empresas clientes de las de seguridad, la subrogación de personal, al transformar los costes fijos asociados al servicio en variables y reducir su riesgo tecnológico, propicia beneficios económicos, organizativos, de continuidad y calidad y de prevención de riesgos laborales en las actividades que le son propias.

También, y como desventaja, resaltar que la acumulación de antigüedad, derivada de las sucesivas subrogaciones, se puede concretar, a efectos indemnizatorios, como un elemento disuasorio de la desvinculación del personal poco comprometido con sus obligaciones laborales.

En definitiva, y como conclusión final, señalar que la subrogación de personal muestra un compendio de ventajas que son dinamizadoras del sector de la seguridad y que se presentan desalentadoras de su supresión o menor regulación.

Para futuras investigaciones, sería deseable que en relación al compromiso de los trabajadores subrogables se realizaran estudios en otras empresas del mismo sector en España, lo que permitiría incrementar la base de información específica del mismo. También, sería deseable comparar dicha información con la que pudiera darse en otros sectores, como los de limpieza de oficinas, viaria, jardinería..., en los que también es de aplicación la figura de la subrogación de personal 


\section{BIBLIOGRAFÍA}

Albrecht, S., y Andreetta, M. (2011). The influence of empowering leadership, empowerment and engagement on affective commitment and turnover intentions in community health service workers: Test of a model. Leadership in Health Services, vol, 24, no 3, pp. 228237. https://doi.org/10.1108/17511871111151126

Allen, N., y Meyer, J. (1990). The measurement and antecedents of affective, continuance and normative commitment to the organization. Journal of Occupational Psychology, vol. 63, no 1, pp. 1-18. https://doi.org/10.1111/j.2044-8325.1990.tb00506.x

Apascaritei, P., y Elvira, M. (2018). Agilidad Estratégica a Través del Capital Humano/ ST479, 1-15. https://doi.org/10.15581/018.ST-479

APROSER. (2018). Información para prensa 2018. Madrid. Retrieved from http://aproser.es/web/wp-content/uploads/2018/01/Informacion-para-Prensa_2018.pdf

Ashman, I., y Winstanley, D. (2006). The ethics of organizational commitment. Business Ethics: A European Review, vol. 15, no 2, pp. 142-153. https://doi.org/10.1111/j.14678608.2006.00438.x

Ávila, S. (2019). Modelo de Gestión del Compromiso Organizacional: Estudio aplicado a una Empresa de Servicios de Seguridad Privada en España. Universidad CEU San Pablo. Retrieved from https://www.educacion.gob.es/teseo/mostrarSeleccion.do

Ávila, S., y Pascual, M. (2019). Propiedades psicométricas de un cuestionario para evaluar la percepción de los trabajadores de las políticas de recursos humanos y su compromiso organizacional. Methaodos. Revista de Ciencias Sociales, vol. 7, n 2, pp. 165-183. https://doi.org/http://dx.doi.org/10.17502/m.rcs.v7i2.258

Ávila, S., y Pascual, M. (2020). Marco filosófico del compromiso organizacional: discusión del modelo de Allen y Meyer y propuesta de un nuevo modelo de estudio. Revista de Estudios Empresariales. Segunda Época., $\mathrm{n}^{\mathrm{o}}$ 1, pp. 201-226. https://doi.org/https://doi.org/10.17561//ree.v2020n1.12

Bauman, Z. (2004). Modernidad líquida. Buenos Aires: Fondo de Cultura Económica de Argentina.

Becker, H. (1960). Notes On the Concept of Commitment. The American Journal of Sociology, vol. 66, n ${ }^{\circ}$ 1, pp. 32-40. https://doi.org/10.1086/222820

Bergman, M. (2006). The relationship between affective and normative commitment: Review and research agenda. Journal of Organizational Behavior, vol. 27, n 5, pp. 645-663. https://doi.org/10.1002/job.372

Bermejo, J. C., Villacieros, M., y Magaña, M. (2014). Perfil, motivación, satisfacción y compromiso de un voluntariado de acompañamiento en duelo. Propiedades psicométricas de los cuestionarios Organizational Commitment Questionnarie y Job Diagnostic Survey. Medicina Paliativa, $\mathrm{n}^{\mathrm{o}}$ 24, pp. 4-13. https://doi.org/10.1016/j.medipa.2014.05.003

Cappelli, P. (2000). The new deal at work. Chicago-Kent Law Review, vol. 76, n² 2, pp. 1169 1193.

Cernas, D. A., y Nava, R. M. (2019). Compromiso organizacional y promedio escolar en estudiantes de maestría: el efecto moderador de las tendencias alocéntricas e idiocéntricas. Revista Electrónica de Investigación Educativa, vol. 21, nº e26, pp. 1-10. https://doi.org/10.24320/redie.2019.21.e26.2122

Cohen, A. (2003). Multiple Commitments in the Workplace: An Integrative Approach. The Academy of Management Review. Psychology Press.

Cohen, A. (2007). Commitment before and after: An evaluation and reconceptualization of organizational commitment. Human Resource Management Review, vol. 17, $\mathrm{n}^{\mathrm{o}} 3$, pp. 336-354. https://doi.org/10.1016/j.hrmr.2007.05.001 
Cooper-Hakim, A., y Viswesvaran, C. (2005). The Construct of Work Commitment: Testing an Integrative Framework. Psychological Bulletin, vol. 131, $\mathrm{n}^{\circ}$ 2, pp. 241-259. https://doi.org/10.1037/0033-2909.131.2.241

Downes, L., y Mui, C. (1999). Estrategias digitales para dominar el mercado. Buenos Aires: Ediciones Granica S.A.

Eulen, S. (2019). La seguridad privada, cada vez más presente. Forbes. Retrieved from https:/forbes.es/empresas/50348/la-seguridad-privada-cada-vez-mas-presente/

EUROPA PRESS. (2019). CC.OO. denuncia un aumento de la conflictividad laboral relacionada con la negociación colectiva. Epeconomía. Retrieved from https://www.europapress.es/economia/laboral-00346/noticia-ccoo-denuncia-aumentoconflictividad-laboral-relacionada-negociacion-colectiva-20190218103737.html

Garrido, E. M. (2016). Compromiso con la profesión docente y con la institución. Cultura docente en la Escuela de Enfermería de la Universidad de Barcelona. De Barcelona. Retrieved from https://www.tesisenred.net/handle/10803/400092\#page=1

González, F., López-Guzmán, T., y Sánchez, S. (2014). Job satisfaction and organizational commitment in human resources in the hotel sector of Cordoba (Spain): Influence of the type of contract and working day. Intangible Capital, vol. 10, n⿳⺈ 1, pp. 189-211. https://doi.org/10.3926/ic.489

González, F., Sánchez, S., y López, T. (2014). Influencia de variables personales en el compromiso organizativo de los empleados del sector hotelero. Revista Galega de Economía, vol. 23, n 2, pp. 27-46.

Haaz, A. (2010). Cadena de valor. Retrieved from https://haazcalidad.blogspot.com/2010/05/cadena-de-valor.html

Hernández, J. G. (2019). La reversión por la administración de las contratas : perspectiva desde el derecho del trabajo Reversion by the administration of the contracts: Gestión y Análisis de Políticas Públicas, $\mathrm{n}^{\mathrm{0}}$ 21, pp. 41-60. https://doi.org/10.24965/gapp.v0i21.10553

Jong-Wook, K., Price, J., y Mueller, C. (1997). Assessment of Meyer and Allen's ThreeComponent Model of Organizational Commitment in South Korea. [Report]. Journal of Applied Psychology December 1997; vol. 82, nº 6, pp. 961-973, vol. 82, nº 6, pp. 961973. https://doi.org/10.1037//0021-9010.82.6.961

Kam, C., Morin, A., Meyer, J., y Topolnytsky, L. (2016). Are Commitment Profiles Stable and Predictable? A Latent Transition Analysis. Journal of Management, vol. 42, n ${ }^{\circ}$ 6, pp. 1462-1490. https://doi.org/10.1177/0149206313503010

La Vanguardia. (2019). La dirección provincial del SAE optimiza el servicio de vigilancia en sus instalaciones. La Vanguardia. Retrieved from https://www.lavanguardia.com/economia/20200309/474049127880/coronavirusespana-economia-plan-medidas-pedro-sanchez.html

Madrigal, M. J., y David, M. (2015). La subrogación de trabajadores y la sucesión de empresa en el ámbito de la contratación pública. Actualidad Jurídica (Uría y Menéndez), no 40, pp. 33-51. Retrieved from https://libros-revistas-derecho.vlex.es/vid/subrogaciontrabajadores-sucesion-empresa-589574102

Mercurio, Z (2015). Affective Commitment as a Core Essence of Organizational Commitment: An Integrative Literature Review. Human Resource Development Review, vol. 14, no 4, pp. 389-414. https://doi.org/10.1177/1534484315603612

Meyer, J., y Allen, N. (1984). Testing the "side-bet theory" of organizational commitment: Some methodological considerations. Journal of Applied Psychology, vol. 69, no 3, pp. 372-378. https://doi.org/10.1037//0021-9010.69.3.372

Moreno-Jiménez, M., y Díaz Gracia, L. (2018). Guía La Prevención de los Riesgos Psicosociales en el sector de las Empresas Multiservicios. 
Morrow, P. (2011). Managing organizational commitment: Insights from longitudinal research. Journal of Vocational Behavior, vol. 79, $\mathrm{n}^{\mathrm{o}}$ 1, pp. 18-35. https://doi.org/10.1016/j.jvb.2010.12.008

Mowday, R., Porter, L., y Dubin, R. (1974). Unit Performance, Situational Factors, and Employee Attitudes in Spatially Separated Work Units. Organizational Behavior and Human Performance, vol. 12, $\mathrm{n}^{\mathrm{o}}$ 2, pp. 231-248. https://doi.org/10.1016/00305073(74)90048-8

Mowday, R., Steers, R., y Porter, L. (1979). The Measurement of Organizational Commitment. Vocational Behavior, nº 14, pp. 224-247. https://doi.org/10.1016/0001-8791(79)900721

Neves, T., Graveto, J., Rodrigues, V., Marôco, J., y Parreira, P. (2018). Organizational commitment, psychometric qualities and invariance of the Meyer and Allen Questionnaire for Portuguese Nurses. Revista Latino-Americana de Enfermagem, vol. 26, no e3021, pp. 1-11. https://doi.org/10.1590/1518-8345.2407.3021

Olea, J., Feuchter, C. I., Barcelo-Valenzuela, M., y Pérez-Soltero, A. (2018). Mejorar la Productividad Mediante la Aplicación de la Curva de Aprendizaje. Retrieved from https://www.researchgate.net/publication/327079572_Mejorar_la_Productividad_Med iante_la_Aplicacion_de_la_Curva_de_Aprendizaje

Pereira, F., Ballarín, E., Rosanas, J. M., y Vázquez-Dodero, J. C. (1989). Contabilidad para dirección. Pamplona-Barcelona: Ediciones Universidad de Navarra, S. A.

Porter, M. (1989). Ventaja Competitiva. Creación y sostenimiento de un desempeño superior (Cuarta). México, D.F.: Compañía editorial continental. https://doi.org/0-02-925090-0

Rivo, E. (1999). Externalización: más allá de la subcontratación. In La gestión de la diversidad. vol. 2, pp. 725-730. Logroño. Retrieved from https://dialnet.unirioja.es/servlet/libro?codigo $=444$

S. Lee, K., y Gao, T. (Tony. (2005). Studying organizational commitment with the OCQ in the Korean retail context: Its dimensionality and relationships with satisfaction and work outcomes. The International Review of Retail, Distribution and Consumer Research, vol. 15, n 4, pp. 375-399. https://doi.org/10.1080/09593960500197495

SECURITAS, G. (2017). Memoria Anual Grupo Securitas. Retrieved from https://www.securitas.es/globalassets/spain/files/memorias-anuales/memoria-anual-securitas-2017.pdf

Swailes, S. (2002). Organizational commitment: A critique of the construct and measures. International Journal of Management Reviews, vol. 4, $\mathrm{n}^{\circ}$ 2, pp. 155-178. https://doi.org/10.1111/1468-2370.00082

UGT. (2020). OMBUDS SERVICIOS | ILUNION OUTSOURCING subroga a los auxiliares de los C.C. CARREFOUR. Retrieved from http://www.fesmcmadrid.org/es/actualidad/ombuds-servicios-ilunion-outsourcingsubroga-a-los-auxiliares-de-los-c-c-carrefour-id-3139.html

Vecina, M. L., y Chacón, F. (2013). Is engagement different from satisfaction and organizational commitment? Relations with intention to remain, psychological wellbeing and perceived physical health in volunteers. Anales de Psicología, vol. 29, $\mathrm{n}^{\circ} 1$, pp. 225-232. https://doi.org/10.6018/analesps.29.1.161861

Yoldi, M. (2019). La seguridad privada, cada vez más presente. Forbes. Retrieved from https://forbes.es/empresas/50348/la-seguridad-privada-cada-vez-mas-presente/ 


\section{ANEXO 1}

ítem COMPROMISO ORGANIZACIONAL. Alfa de Cronbach 0,92

1 Cuando hablo con mis amigos les digo que mi organización es un gran lugar para trabajar.

2 Soy leal a esta organización.

3 Sí que merece la pena permanecer trabajando en esta organización indefinidamente.

4 Estoy dispuesto a realizar un gran esfuerzo para contribuir al éxito de la organización.

5 Definitivamente, la decisión de trabajar en esta organización ha sido un acierto por mi parte.

6 Me importa mucho el futuro de esta organización.

7 Para mí esta es la mejor organización posible para trabajar. 